\title{
REDUCTION OF ENDOTHELIAL INJURY AFTER HYPOTHERMIC LUNG PRESERVATION BY INITIAL LEUKOCYTE-DEPLETED REPERFUSION
}

\author{
A. J. Levine, BSc, FRCS (CTh) \\ K. Parkes \\ S. Rooney, BmedSci, FRCS \\ R. S. Bonser, BSc, MRCP, FRCS
}

Objectives: Leukocyte depletion has been shown to ameliorate the effects of reperfusion injury in many organ systems. The aim of this study was to investigate the effects of leukocyte depletion on functional and endothelial markers of pulmonary performance after cold ischemic injury.

Method: Groups of 6 rat lungs were flushed with University of Wisconsin solution and then stored at $4^{\circ} \mathrm{C}$ for 4 hours. They then underwent sanguine reperfusion for 30 minutes, during which time functional measures (gas exchange, pulmonary artery, and airway pressures) were made and after which the lungs underwent estimation of endothelial permeability by measurement of the capillary filtration coefficient (in grams per centimeter of water per minute per grams of wet lung tissue) by a gravimetric technique. Four groups were studied: group 1 underwent no reperfusion, group 2 underwent 30 minutes of reperfusion, group 3 underwent 30 minutes of leukocytedeplete reperfusion with an in-line leukocyte filter (PALL), and group 4 underwent 10 minutes of leukocyte-depleting reperfusion followed by 20 minutes of normal reperfusion.

Results: The capillary filtration coefficient increased between group 1 and group 2 animals $(1.05 \pm 0.32$ to $3.07 \pm 0.47$ [mean $\pm \mathrm{SEM}] ; P<.01)$. Complete leukocyte depletion caused the greatest diminution in the capillary filtration coefficient $(0.392 \pm 0.07, P<.001)$, but initial leukocyte depletion (group 4) also showed a significant diminution $(0.74 \pm 0.3, P<.01)$. Complete or initial leukocyte depletion caused no significant change in functional measures of pulmonary performance. Complete leukocyte depletion produced less pulmonary leukostasis, as assessed by means of myeloperoxidase activity.

Conclusion: Initial and continued leukocyte depletion are associated with amelioration of reperfusion-induced endothelial injury after cold ischemic injury. (J Thorac Cardiovasc Surg 2000;120:47-54)
$P^{\prime}$ rimary graft failure is a common cause of mortality and morbidity after clinical lung transplantation. ${ }^{1} \mathrm{It}$ is mainly due to reperfusion injury and is manifested as

From the Cardiothoracic Surgical Unit, Queen Elizabeth Hospital, Edgbaston, Birmingham, United Kingdom.

Received for publication June 22, 1999; revisions requested Sept 16, 1999; revisions received Jan 10, 2000; accepted for publication March 10, 2000.

Address for reprints: R. S. Bonser, Cardiothoracic Surgical Unit, Queen Elizabeth Hospital, Edgbaston, Birmingham, B15 2TH, United Kingdom (E-mail: r.s.bonser@ bham.ac.uk).

Copyright (c) 2000 by The American Association for Thoracic Surgery

$0022-5223 / 2000 \$ 12.00+0 \quad \mathbf{1 2 / 1 / 1 0 7 1 2 6}$

doi: $10.1067 / \mathrm{mtc} .2000 .107126$ a mixed vascular and endothelial injury. ${ }^{2}$ Although reperfusion injury is a complex phenomenon, the neutrophil plays a central role in its cause and modulation. Leukocyte depletion has been shown to ameliorate reperfusion injury in the heart and the lungs. ${ }^{3-14}$ Despite evidence of a beneficial effect of leukocyte depletion in laboratory studies, the limited efficacy of currently available mechanical filters and concerns of infective complications in immunocompromised patient have prevented clinical studies in lung transplantation.

Most studies of leukocyte depletion have investigated warm ischemia or long cold ischemic periods, and it is not known whether the benefits are applicable to the shorter cold ischemic times (4-6 hours) seen in clinical practice. ${ }^{15}$ Moreover, we hypothesized that a short ini- 


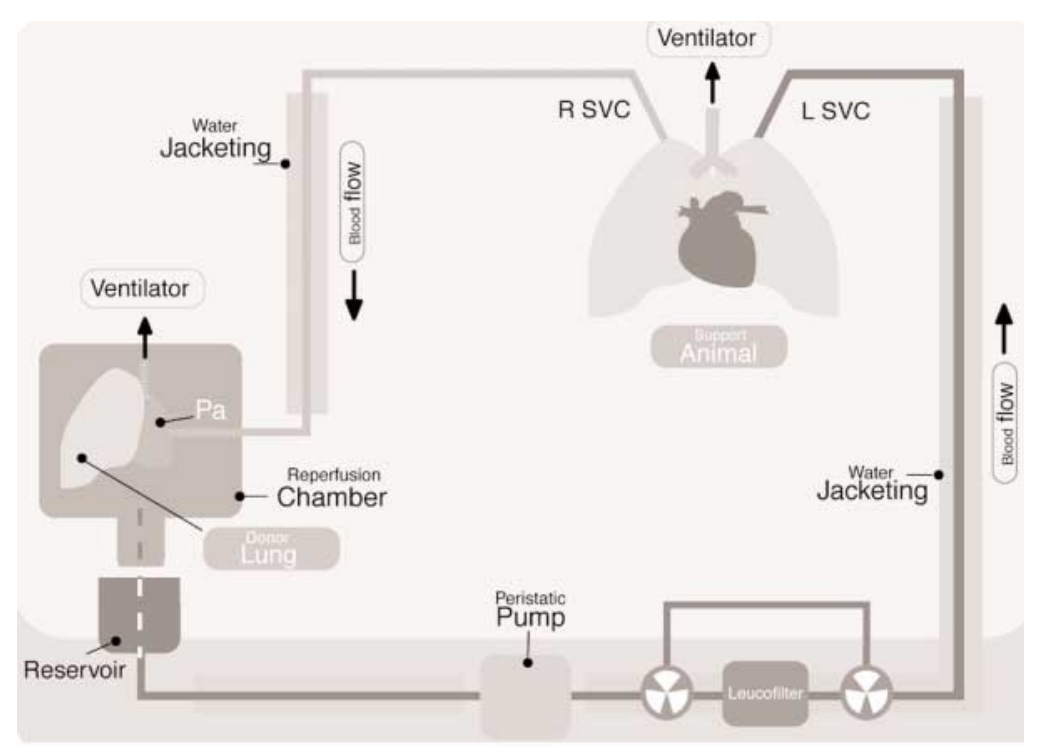

Fig 1. Schematic diagram of leukocyte-depletion reperfusion circuit.

tial period of leukocyte-depleting reperfusion would have a beneficial effect equivalent to that of continued leukocyte-depleting reperfusion after a period of hypothermic lung preservation.

\section{Methods}

Isolated rat lung grafts were reperfused in a circuit by use of a parabiotic animal. ${ }^{16}$ During reperfusion, functional indices of graft performance (gas exchange, airway pressure, and pulmonary artery pressure) were measured, and at the end of the reperfusion period, the capillary filtration coefficient, ${ }^{17}$ wet/dry weight ratios, and myeloperoxidase activity were assessed.

Male Sprague-Dawley rats (Charles River Laboratories, Kent, United Kingdom) weighing 450 to $600 \mathrm{~g}$ were used. All animals received humane care in compliance with the United Kingdom Government's Animals (Scientific Procedures) Act of 1986, and all procedures were performed with the use of terminal anesthesia. Halothane and oxygen inhalation and intraperitoneal ketamine/metatomadine were used for anesthetic induction and maintenance, respectively. Animal lungs and isolated lung grafts were ventilated with the use of Harvard rodent ventilators (Harvard Apparatus, Kent, United Kingdom).

Graft harvest. After anesthesia, tracheostomy was performed with a 13-gauge venous cannula, and the animal's lungs were ventilated with a tidal volume of $10 \mathrm{~mL} / \mathrm{kg}$, a rate of 70 breaths $/ \mathrm{min}$, and $2 \mathrm{~cm}$ of positive end-expiratory pressure. Median sternotomy was carried out followed by division of pleura and pericardium and retraction of the thymus. The aorta and pulmonary artery were then encircled with a ligature passed through the transverse sinus. Heparinization (1000 $\mathrm{U} / \mathrm{kg}$ ) was performed through the inferior vena cava, which was then ligated to diminish venous return. An incision was made in the right ventricular outflow tract, and a spiggoted arteriotomy cannula was advanced into the pulmonary artery and secured with the transverse sinus ligature. The left ventricular apex was then amputated to allow venting. University of Wisconsin solution (DuPont Pharmaceuticals, Letchworth Garden City, United Kingdom) was then infused at a dose of $60 \mathrm{~mL} / \mathrm{kg}$ at $4^{\circ} \mathrm{C}$ under $20 \mathrm{~cm} \mathrm{H}_{2} \mathrm{O}$ pressure. Ventilation continued while the flush was completed, at which point the tracheostomy tube was clamped with the lungs fully inflated. The spigoted cannula was then changed for an appropriately primed and amputated triple-lumen catheter. The heart-lung block was then excised and stored in University of Wisconsin solution at $4^{\circ} \mathrm{C}$ for 4 hours. Control blocks then had a second amputated triple-lumen catheter placed in the left atrium through the ventriculotomy. Reperfusion blocks had this cannula inserted after their period of reperfusion.

Reperfusion. Support animals were anesthetized and ventilated and placed on a homeothermic warming blanket. After median sternotomy and pleural and pericardial division, the left superior vena cava (SVC) was cannulated with an 18gauge cannula. The right SVC was cannulated with a 16gauge cannula that was passed through the right atrium into the suprahepatic inferior vena cava. Deoxygenated blood drawn through the right SVC cannula was delivered hydrostatically into the pulmonary artery of the donor block through the triple-lumen catheter. The blood then drained through the left ventriculotomy and was collected and returned by using a peristaltic pump (Variable speed peristaltic pump, Harvard Apparatus) through the left SVC cannula to the right atrium of the support animal. The support animal and donor block were ventilated in the same manner as the donor animal. The extracorporeal circuit consisted of an inner blood-carrying tube encased in an outer warming jacket with a countercurrent 


\section{Change in gas exchange leukocyte deplete reperfusion v. normal reperfusion}

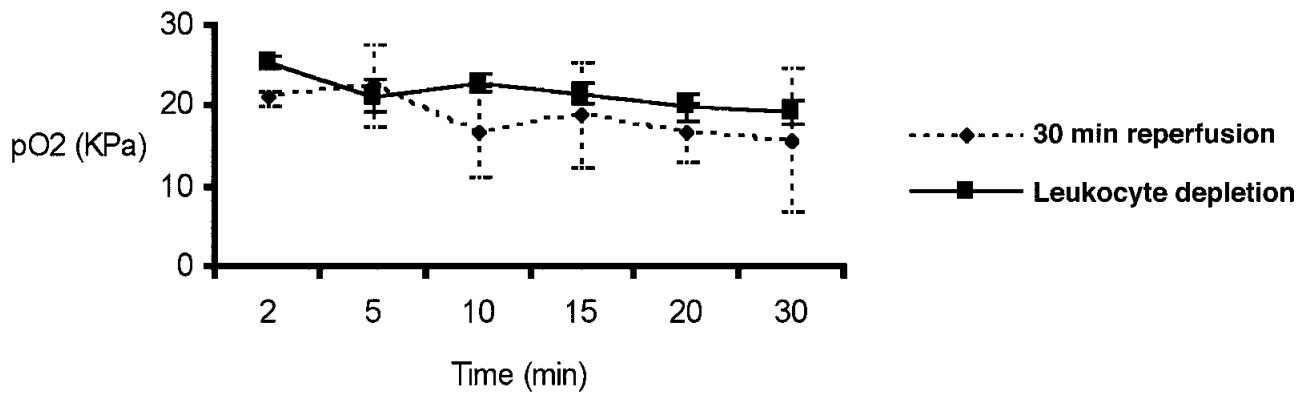

\section{Change in airway pressure (AWP) leukocyte deplete reperfusion v. normal} reperfusion

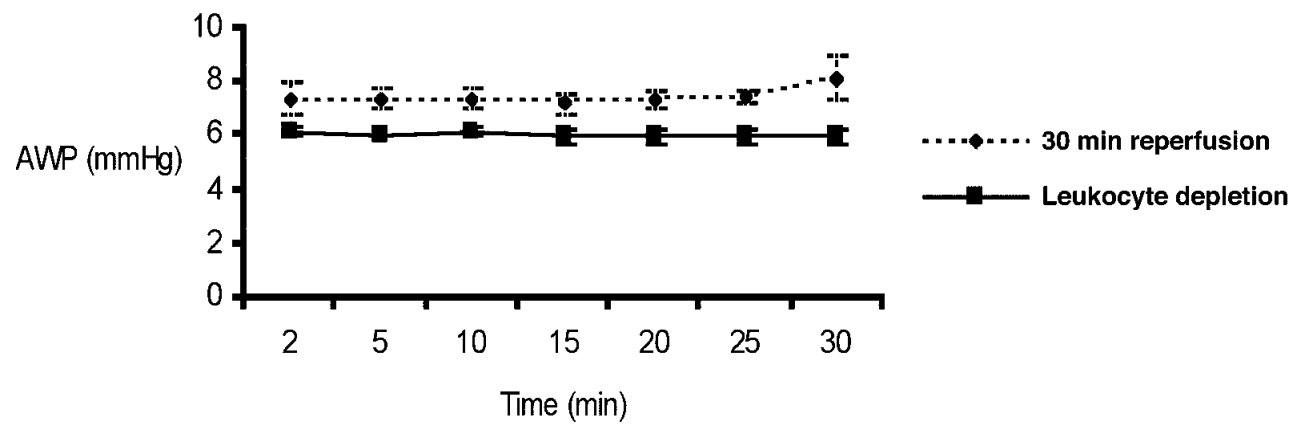

\section{Change in pulmonary artery pressure (PAP) leukocyte deplete reperfusion v. normal reperfusion}

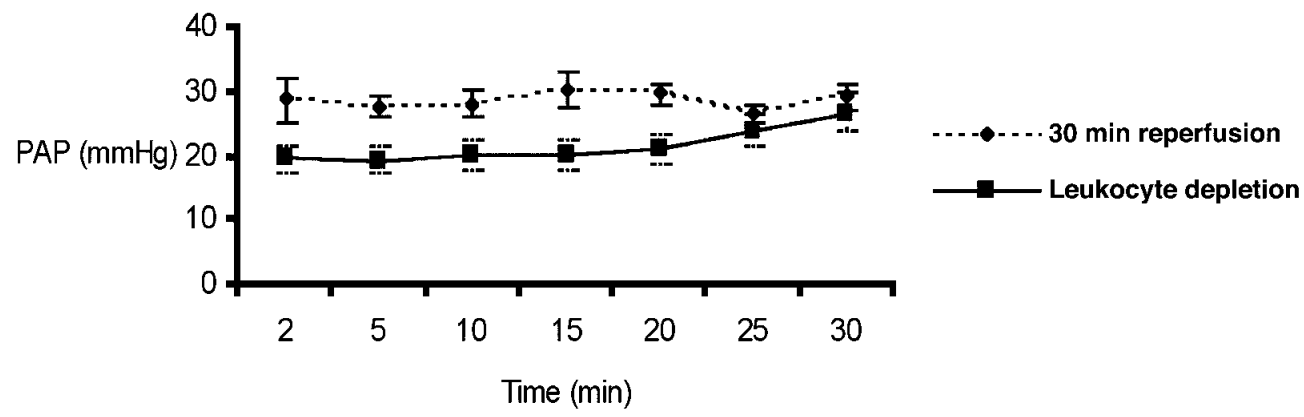

Fig 2. Full leukocyte depletion. Results are expressed as means \pm SEM.

$38^{\circ} \mathrm{C}$ water circulation circuit. The lung block was mounted in a Plexiglas (Rohm and Haas Company, Philadelphia, Pa) perfusion chamber with an outer warming jacket. The circuit was primed with heparinized blood from a further 2 animals and maintained air and bubble free.

Leukocyte-depleting reperfusion. Leukocyte-depleting reperfusion was achieved by the use of an in-line PALL J1179 leukofilter (Pall Europe, Portsmouth, United
Kingdom). This was placed in circuit between the block effluent and the cannula into the support animal left SVC distal to the peristaltic pump (Fig 1). It was placed here to facilitate an adequate pressure through the filter to allow filtration with associated leukocyte depletion. A bypass circuit was placed around the filter to allow the filter to be in-line or offline in the circuit. Thus in the initial 10-minute, leukocytedepleted, reperfusate series, the filter was left in-line for the 
initial 10-minute period and then placed off-line for the last 20 minutes. The circuit was fully primed with leukocytedepleted blood.

Measurements. Graft effluent was sampled at 2, 5, 10, 15, 20 , and 30 minutes for gas tension. The pulmonary artery pressure was measured through a limb of the triple-lumen catheter, as was the airway pressure through the tracheostomy tube.

Two hundred-microliter samples of blood were taken for estimation of white cell number from 3 points in the circuit:

1. Proximal to the donor block (position A)

2. Proximal to the filter (position B)

3. Distal to the filter but proximal to the left SVC cannula (position C)

Samples were taken at $0,5,10,15$, and 30 minutes during the reperfusion period.

The white blood cell number was estimated by using a Sysmex NE8000 (Sysmex Corporation of America, Long Grove, Ill).

Capillary filtration coefficient. A modification of the method of Drake and colleagues ${ }^{17}$ was used to assess the capillary filtration coefficient $\left(\mathrm{K}_{\mathrm{fc}}\right)$. This is a gravimetric technique in which $\mathrm{K}_{\mathrm{fc}}$ is calculated from the plot of the rate of lung weight gain as a function of hydrostatic stress placed on the pulmonary vasculature. By using a nonhemic but physiologic iso-osmotic perfusate of L15 (Sigma Chemical Co, St Louis, Mo) and fetal calf serum in the ratio of 9:1, a hydrostatic stress was applied to the isolated lung under test by increasing the pulmonary artery pressure. On application of a known hydrostatic pressure, there was initially a short period of rapid weight gain attributable to recruitment and cardiac filling. Subsequently, a slow weight gain phase occurred, and by extrapolating the slope of this rate of weight gain to time zero, the initial rate of fluid flux was estimated. $\mathrm{K}_{\mathrm{fc}}$ can be calculated from this initial rate of fluid flux by application of the Starling-Landis equation.

Postcaval lobes of ischemic or reperfused heart-lung blocks were identified, ligated, and removed for estimation of wet/dry ratio and myeloperoxidase assay. The block was suspended from a force transducer (Harvard Apparatus) with continuous weight monitoring by using a pen recorder (Harvard Apparatus) through a 3-gauge stainless steel wire passed through the esophagus, and ventilation was maintained as previously. The pulmonary artery and the left atrial cannulas were connected in turn. A pressure of $4 \mathrm{~mm} \mathrm{Hg}$ was applied to the arterial cannula by elevation of the L15/fetal calf serum reservoir. Equilibration of the circuit was assessed to have occurred when the left atrial pressure matched the pulmonary artery pressure, thus equaling the pulmonary capillary pressure. At this point, the arterial pressure was raised to $5 \mathrm{~mm} \mathrm{Hg}$, and the weight gain trace was recorded for a 10 minute period. The arterial pressure was finally raised to 7 $\mathrm{mm} \mathrm{Hg}$, and the trace was recorded for a further 10-minute period. The blocks were then taken down, and the parenchymal lung tissue excised for estimation of wet/dry ratio by desiccation (Pearce Edwards Freeze Drier EPTD3; Edwards, Sussex, United Kingdom).
Myeloperoxidase assay. Myeloperoxidase occurs mainly in the primary granules of neutrophils, and it is used as a quantitative marker of neutrophil sequestration in pulmonary tissue. A modification of the assay of Goldblum and colleagues $^{18}$ was used to assess myeloperoxidase activity. Samples of the postcaval lobe were taken, snap frozen in liquid nitrogen, and stored at $-70^{\circ} \mathrm{C}$ until analysis. Fifty-milligram pieces of tissue were homogenized with $200 \mu \mathrm{L}$ of water to give a 1:5 dilution; this was further diluted with water to a 1:25 dilution, and the resulting suspension was centrifuged at $5000 \mathrm{rpm}$ for 5 minutes. Twenty-microliter samples of the supernatant were added to $180 \mu \mathrm{L}$ of substrate (containing $0.167 \mathrm{mg} / \mathrm{mL} \sigma$-dianisidine $2 \mathrm{HCl}$ and $0.0005 \%$ $\mathrm{H}_{2} \mathrm{O}_{2}$ [final volume, $3 \mathrm{ml}$ of 1:30 dilution]) in wells and left for 15 minutes, after which absorbance was read at $472 \mathrm{~nm}$ on an enzyme-linked immunosorbent assay reader. Synchronously standard curves were prepared by using known quantity of myeloperoxidase (Sigma Chemical Co) where 1 unit produced an increase in absorbance $\left(\Delta \mathrm{A}_{472}\right)$ of 1 per minute.

Myeloperoxidase activity in the samples was calculated from their relative change in absorbance and the known activity of the enzyme standard.

Experimental protocol. Four groups of randomly assigned lung blocks $(n=6)$ were used. Group 1 (control) underwent flush, excision, storage for 4 hours, and measurement of filtration coefficient. Group 2 (normal reperfusion) underwent flush, excision, storage, and 30 minutes of nonleukocyte-depleting reperfusion, followed by measurement of filtration coefficient. Group 3 (complete leukocyte-depleting reperfusion) underwent flush, excision, storage, and 30 minutes of reperfusion with the filter in-line, followed by measurement of filtration coefficient. Group 4 (initial leukocyte-depleting reperfusion) underwent flush, excision, storage, and 10 minutes of reperfusion with the filter in-line, followed by 20 minutes of reperfusion with the filter off-line. After this second period, measurement of the filtration coefficient occurred.

Statistical analysis. Data are expressed as mean values \pm SEM. Group means of final measurements were compared by using analysis of variance (Newman-Keuls). Analysis of repeated measures was carried out by multiple analysis of variance. Analysis was carried out by SPSS software (SPSS Inc, Chicago, Ill).

\section{Results}

Capillary filtration coefficient. There was a significant rise of capillary filtration coefficient between groups 1 and $2(1.048 \pm 0.316$ to $3.063 \pm 0.466$, $P<.01)$ demonstrating reperfusion-induced hyperpermeability. This effect was ameliorated by complete leukocyte-depleting reperfusion $(0.392 \pm 0.027, P<.001)$ and by initial leukocyte-depleting reperfusion $(0.735 \pm 0.121$, $P<.01$ ), but there was no significant difference between the two leukocyte-depleting strategies. 


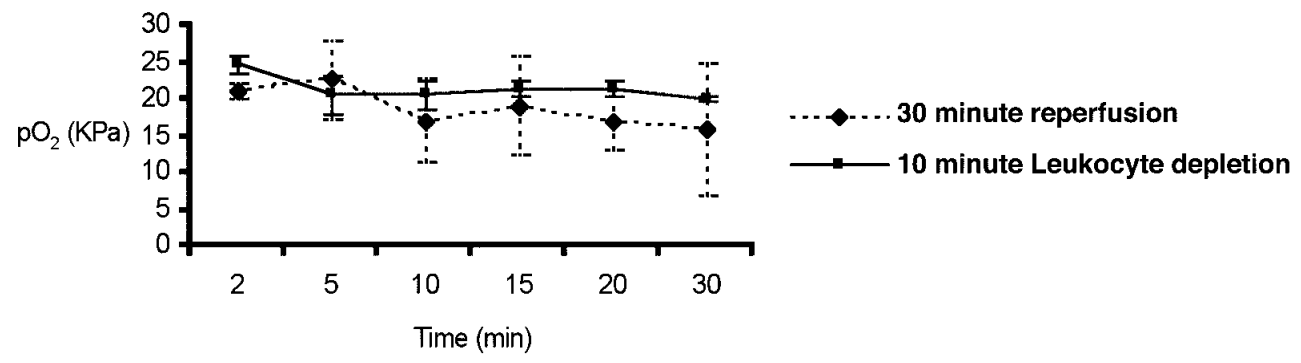

Change in airway ressure (AWP) with an initial 10 minute leukocyte deplete sanguineous reperfusate v. normal reperfusion

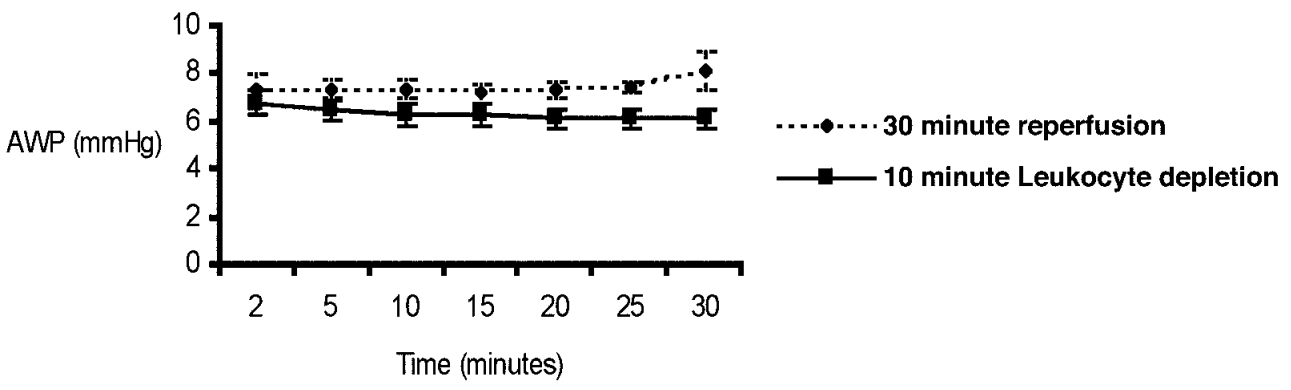

Change in pulmonary artery pressure (PAP) with an initial 10 minute leukocyte deplete sanguineous reperfusate v. normal reperfusion

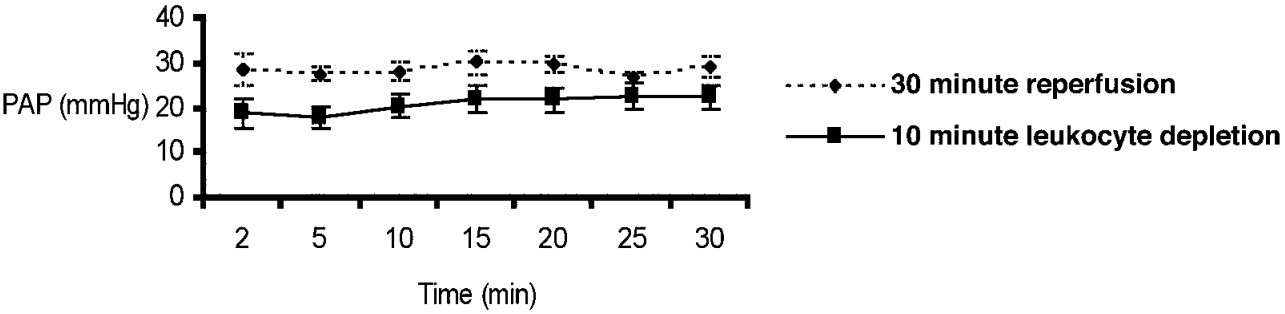

Fig 3. Initial leukocyte depletion. Results are expressed as means \pm SEM.

Functional measures. The functional data (Figs 2 to 4) suggest that there were no significant differences with respect to functional measures between the normally reperfused, the leukocyte-depleted reperfused, and the initially leukocyte-depleted reperfused groups.

Wet/dry ratio. There was no significant difference between the wet/dry ratios of groups 2 and 3 (5.653 \pm 0.124 to $5.205 \pm 0.169, P=.47)$ nor any between groups 2 and 4 (5.374 $\pm 0.402, P=.42)$.

White blood cell count. The absolute leukocyte number of group 3 experiments at position A (Fig 1) climbed to a plateau after 5 minutes (Fig 4). The efficiency of extraction of leukocytes (defined as the absolute leukocyte number at B/absolute leukocyte number at $\mathrm{C}$ ) increased with time, suggesting that the PALL filter was working efficiently (Fig 5).

Myeloperoxidase assay. There was a significant diminution in myeloperoxidase activity between groups 2 and $3(50.9 \pm 11.3$ to $28.6 \pm 6.4, P<.05)$, demonstrating reduced pulmonary leukostasis after continued leukocyte depletion. There was a reduction in myeloperoxidase activity in group $4(31.3 \pm 4.9, P=$ $.25)$, but this did not reach significance. 


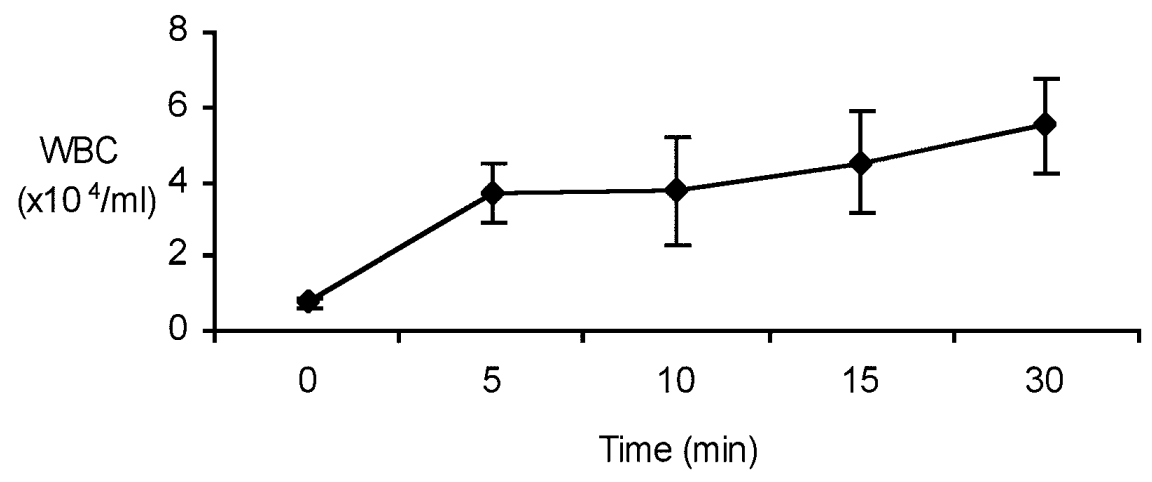

Fig 4. Change in absolute leukocyte number with time at the donor lung block.

\section{Discussion}

This study demonstrates that increased endothelial permeability seen after 30 minutes of reperfusion after 4 hours of hypothermic preservation is reduced by leukocyte-depleting reperfusion. This endothelial protection is despite the relative inefficiency of complete leukocyte depletion by the mechanical filter. Moreover, a 10-minute initial period of leukocyte-depleting reperfusion seems to offer a similar degree of protection as that offered by complete leukocyte-depleting reperfusion against this endothelial damage. At this preservation period, complete leukocyte depletion produces less pulmonary leukostasis than normal sanguineous reperfusion.

Neutrophil-derived effector mechanisms are central to the cause of reperfusion injury in many organ systems. ${ }^{19-22}$ Because of this role, leukocyte depletion has been investigated as a means of ameliorating reperfusion injury in the heart ${ }^{3,4}$ and has been found to be clinically effective..$^{23-25}$ Leukocyte depletion has also been shown to reduce pulmonary injury associated with cardiopulmonary bypass, ${ }^{8,26}$ although less efficient methods of leukocyte depletion have yet to demonstrate efficacy. ${ }^{27}$

Leukocyte depletion is very difficult to achieve. Seibert and colleagues ${ }^{14}$ demonstrated that even lungs undergoing continued nonhemic perfusion contained significant numbers of endogenous neutrophils. It is also possible that the role of sequestered neutrophils in the lung may be different than that in other organs. ${ }^{28}$ The role of leukocyte depletion in ameliorating pulmonary injury is controversial because injury can also occur in their absence. However, several studies have demonstrated that leukocyte depletion can ameliorate pulmonary reperfusion injury. ${ }^{5-14}$ Eppinger and col- league ${ }^{29}$ have suggested that there is a biphasic pattern to endothelial damage with respect to pulmonary injury and that the early part of this is independent of neutrophil participation. Further studies with a warm ischemic model reperfused with an asanguineous medium (PSS-Ficoll) have suggested that neutrophils play no part in the evolution of reperfusion-induced endothelial damage. ${ }^{28}$

Conversely, lung transplant recipient animals immunodepleted of neutrophils before transplantation demonstrate improved graft function. ${ }^{5}$ Mechanical leukocyte depletion has also been shown to diminish free radical activity and histologic injury after a 2hour warm ischemic insult. ${ }^{6,7}$ Other workers ${ }^{8,9}$ have noted the beneficial effect of an in-line filter in a cardiopulmonary bypass circuit in relation to pulmonary function. Ross and colleagues ${ }^{30}$ in a novel rabbit 18 hour preservation model noted that efficient mechanical leukocyte depletion resulted in less microvascular injury with increased functional performance and less neutrophil sequestration, although the method of assessing microvascular permeability has been questioned. ${ }^{31}$ Finally, a number of studies have demonstrated the beneficial effects of leukocyte depletion in models of lung and heart-lung transplantation. ${ }^{10-13}$ Schueler and colleagues, ${ }^{10}$ in a bovine double lung transplant model with donor and recipient mechanical leukocyte depletion, demonstrated improved lung function (as defined by histologic and functional criteria) after 24 hours of ischemia. Pillai, ${ }^{11}$ Breda, ${ }^{12}$ Bando, ${ }^{13}$ and their colleagues, all using heart-lung blocks, similarly found better pulmonary function after variable periods of cold ischemia (12-24 hours) with leukocyte-depleted reperfusate. This study demonstrates that 10 minutes of leukocyte-depleting 


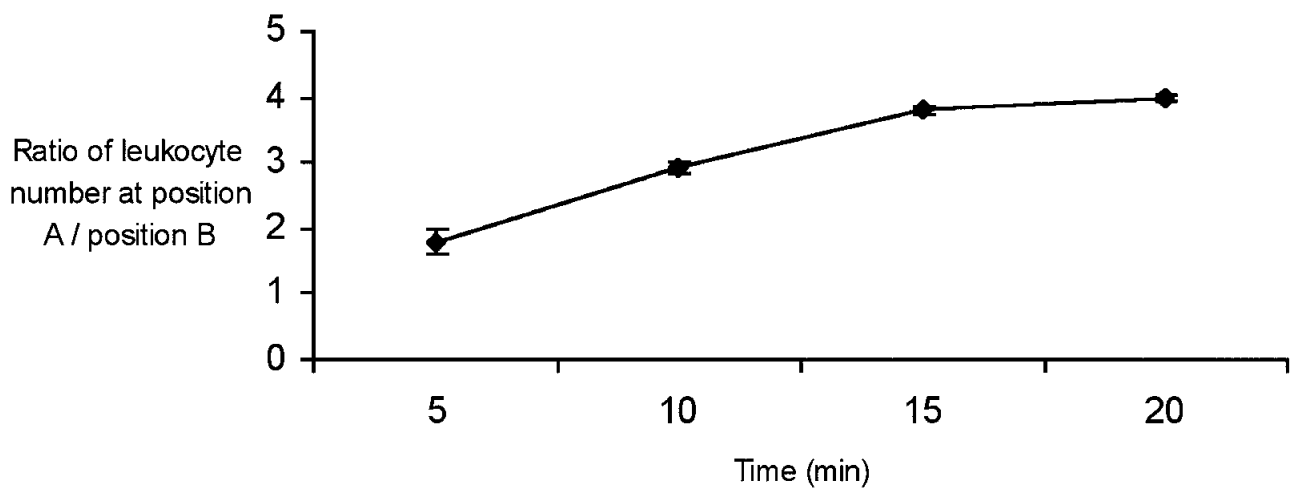

Fig 5. Efficiency of leukocyte extraction by filter with time during reperfusion.

reperfusion confers similar endothelial protection as prolonged leukocyte-depleting reperfusion. This finding has been previously demonstrated in the heart ${ }^{4}$ but not, to our knowledge, in the lung. The initial reperfusion of an implanted organ for a 10-minute period by use of a partially leukocyte-depleted source of blood is clinically feasible and counters any objections of whole body leukocyte depletion in the immunocompromised transplant recipient.

Leukocyte depletion by means of mechanical filtration is difficult to attain in any sanguineous circuit connected to a support animal or in the clinical situation. Where the circuit is not connected to a support animal, very considerable leukocyte depletion can occur, down to $0.6 \%$ of normal levels. ${ }^{30}$ However, in situations where recruitment of leukocytes can occur from a number of sources, including the lung and bone marrow, it is not possible to attain such levels. The degree of leukocyte depletion in this group of experiments can be seen to be analogous to levels seen in clinical leukocyte depletion during cardiopulmonary bypass. ${ }^{26,27,32}$

The ability of sustained leukocyte depletion to reduce reperfusion-induced hyperpermeability is intuitive considering the important role of the neutrophil in reperfusion injury. However, it is more difficult to understand the mechanism of initial leukocyte-depleting reperfusion. It is possible that maximal neutrophil-related injury occurs early after reperfusion but that the consequences of this injury are not evident until a later time. ${ }^{4}$ It might also be possible that susceptibility of the endothelium to neutrophil-mediated injury diminishes over time. This was not investigated in this group of experiments and would be difficult to study in any parabiotic model that necessitated the exposure of blood to foreign surfaces. Although initial and sustained leukocyte depletion did not affect simple func- tional measurements, this may have been due to the limited study period. ${ }^{33}$

Initial and prolonged leukocyte-depleting reperfusion of hypothermically preserved lung grafts reduces endothelial injury and, in the case of prolonged depletion, reduces pulmonary neutrophil infiltration. These findings demonstrate the importance of the neutrophil in the cause and modulation of pulmonary reperfusion injury. Initial leukocyte depletion has clinical potential in the prevention of primary graft failure.

We thank Dr P. Davies (Department of Statistics, University of Birmingham, United Kingdom) for kind assistance in the statistical analysis of this work.

\section{REFERENCES}

1. Bando K, Paradis IL, Komatsu K, et al. Analysis of time-dependent risks for infection, rejection, and death after pulmonary transplantation. J Thorac Cardiovasc Surg 1995;109:49-57.

2. Pinsky DJ. The vascular biology of heart and lung preservation for transplantation. Thromb Haemost 1995;74:58-65.

3. Fukushima N, Shirakura R, Nakata S, et al. Effects of terminal cardioplegia with leukocyte-depleted blood on heart grafts preserved for 24 hours. J Heart Lung Transplant 1992;11:676-82.

4. Galinanes M, Lawson C, Ferrari R, Limb G, Derias N, Hearse D. Early and late effects of leukopenic reperfusion on the recovery of cardiac contractile function-studies in the transplanted and isolated blood perfused rat heart. Circulation 1993;88:673-83.

5. Naka Y, Toda K, Kayano K, Oz MC, Pinsky DJ. Failure to express the P-selectin gene or P-selectin blockade confers early pulmonary protection after lung ischemia or transplantation. Proc Natl Acad Sci U S A 1997;94:757-61.

6. Ide H, Ino T, Hasegawa T, Matsumoto H. The role of leukocyte depletion by in vivo use of leukocyte filter in lung preservation after warm ischemia. Angiology 1990;41:318-27.

7. Ide $\mathrm{H}$, Ino $\mathrm{T}$, Hasegawa $\mathrm{T}$, Matsumoto $\mathrm{H}$. The effect of peripheral blood leukocyte depletion on reperfusion injury of warm ischemic lung. Nippon Geka Gakkai Zasshi 1990;91:1023-30.

8. Bando K, Pillai R, Cameron DE, et al. Leukocyte depletion ame- 
liorates free radical-mediated lung injury after cardiopulmonary bypass. J Thorac Cardiovasc Surg 1990;99:873-7.

9. Hachida M, Hanayama N, Okamura T, et al. The role of leukocyte depletion in reducing injury to myocardium and lung during cardiopulmonary bypass. ASAIO J 1995;41:M291-4.

10. Schueler S, De Valeria PA, Hatanaka M, et al. Successful twentyfour-hour lung preservation with donor core cooling and leukocyte depletion in an orthotopic double lung transplantation model. J Thorac Cardiovasc Surg 1992;104:73-82.

11. Pillai R, Bando K, Schueler S, Zebly M, Reitz BA, Baumgartner WA. Leukocyte depletion results in excellent heart-lung function after 12 hours of storage. Ann Thorac Surg 1990;50:211-4.

12. Breda MA, Hall TS, Stuart RS, et al. Twenty-four hour lung preservation by hypothermia and leukocyte depletion. J Heart Transplant 1985;4:325-9.

13. Bando K, Schueler S, Cameron DE, et al. Twelve-hour cardiopulmonary preservation using donor core cooling, leukocyte depletion, and liposomal superoxide dismutase. J Heart Lung Transplant 1991;10:304-9.

14. Seibert AF, Haynes J, Taylor A. Ischemia-reperfusion injury in the isolated rat lung. Role of flow and endogenous leukocytes. Am Rev Respir Dis 1993;147:270-5.

15. Hopkinson DN, Bhabra MS, Hooper TL. Pulmonary graft preservation: a worldwide survey of current clinical practice. J Heart Lung Transplant 1998;17:525-31.

16. Hopkinson DN, Odom NJ, Eloakley RM, Au J, Hooper TL. A technique for studying the function of an isolated, perfused and ventilated lung in a rat model. Lab Anim 1995;29:96-101.

17. Drake R, Gaar KA, Taylor AE. Estimation of the filtration coefficient of pulmonary exchange vessels. Am $\mathrm{J}$ Physiol 1978;234:H266-74.

18. Goldblum SE, Wu KM, Jay M. Lung myeloperoxidase as a measure of pulmonary leukostasis in rabbits. J Appl Physiol 1985;59:1978-85.

19. Brophy CM. Gastrointestinal vascular and ischemic syndromes. Curr Opin Gen Surg 1993;15:225-31.

20. Weight SC, Bell PR, Nicholson ML. Renal ischaemia-reperfusion injury. Br J Surg 1996;83:162-70.

21. Williams FM. Neutrophils and myocardial reperfusion injury. Pharmacol Ther 1996;72:1-12.
22. Thiagarajan RR, Winn RK, Harlan JM. The role of leukocyte and endothelial adhesion molecules in ischemia-reperfusion injury. Thromb Haemost 1997;78:310-4.

23. Sawa Y, Taniguchi K, Kadoba K, et al. Leukocyte depletion attenuates reperfusion injury in patients with left ventricular hypertrophy. Circulation 1996;93:1640-6.

24. Pearl J, Drinkwater D, Laks H, Capouya E, Gates R. Leukocytedepleted reperfusion of transplanted human hearts: a randomized, double-blind clinical trial. J Heart Lung Transplant 1992;11:1082-92.

25. Pearl J, Drinkwater D, Laks H, Stein D, Capouya E, Bhuta S. Leukocyte-depleted reperfusion of transplanted human hearts prevents ultrastructural evidence of reperfusion injury. J Surg Res 1992;52:298-308.

26. Gu YJ, de Vries AJ, Boonstra PW, van Oeveren W. Leukocyte depletion results in improved lung function and reduced inflammatory response after cardiac surgery. J Thorac Cardiovasc Surg 1996;112:494-500.

27. Gu YJ, Vries Ad, Paulien V, Boonstra PW, Oeveren Wv Leukocyte depletion during cardiac operation: a new approach through the venous bypass circuit. Ann Thorac Surg 1999;67:604-13

28. Deeb GM, Grum CM, Lynch MJ, et al. Neutrophils are not necessary for induction of ischemia-reperfusion lung injury. J Appl Physiol 1990;68:374-81.

29. Eppinger MJ, Deeb GM, Bolling SF, Ward PA. Mediators of ischemia-reperfusion injury of rat lung. Am $\mathrm{J}$ Pathol 1997;150:1773-84.

30. Ross S, Tribble C, Gaughen J, Shockey K, Parrino P, Kron I. Reduced neutrophil infiltration protects against lung reperfusion injury after transplantation. Ann Thorac Surg 1999;67:1428-35.

31. Dallal MM, Chang SW. Evans blue dye in the assessment of permeability-surface are product in perfused rat lungs. J Appl Physiol 1994;77:1030-5.

32. Lust RM, Bode AP, Yang L, Hodges W, Chitwood WR Jr. In-line leukocyte filtration during bypass. Clinical results from a randomized prospective trial. ASAIO J 1996;42:M819-22.

33. Rooney S, Farneti P, Shimada I, Bonser R. Increasing pulmonary capillary permeability after ischaemic preservation is not detected by functional lung assessment. Heart 1998;79(Suppl 1):29. 